\title{
Determinants of Surgical Case On-Time Start and On-Time Finish in Perioperative Services
}

\author{
Majbah Uddin ${ }^{1, *}$, Lawrence D. Fredendall ${ }^{2}$, Nathan Huynh ${ }^{3}$, Kevin M. Taaffe ${ }^{4}$, and Robert \\ Allen 5 \\ 1 Civil and Environmental Engineering Department, University of South Carolina, Columbia, SC, USA; \\ muddin@mailbox.sc.edu \\ 2 Management Department, Clemson University, Clemson, SC, USA; flawren@clemson.edu \\ 3 Civil and Environmental Engineering Department, University of South Carolina, Columbia, SC, USA; \\ nathan.huynh@sc.edu \\ 4 Industrial Engineering Department, Clemson University, Clemson, SC, USA; taaffe@clemson.edu \\ 5 Perioperative Services, Prisma Health - Upstate Affiliate, Greenville, SC, USA; \\ Robert.Allen@prismahealth.org \\ * Correspondence: muddin@mailbox.sc.edu
}

\begin{abstract}
Efficient use of the operating room (OR) is crucial for any hospital. One of the major inefficiencies in the OR is surgical cases not starting or finishing on time as scheduled. When a case is delayed, it affects all subsequent cases in that OR. This study uses discrete choice analysis to determine the significant factors, including team familiarity, that influence OR case on-time start and finish. A case is considered on-time if the documented procedure start and finish times are no more than 10 minutes after the scheduled start and finish times. The analysis uses surgical case data from a large tertiary referral hospital and academic center in Greenville, South Carolina. The case data includes all surgical cases $(15,091)$ performed during regular workdays in 2013 . Two binary logit models are developed: one for case on-time start and one for case on-time finish. Results indicate that higher team familiarity between surgeon and anesthesiologist, surgeon and circulating nurse, surgeon and scrub nurse, and surgeon and CRNA improve the likelihood of an OR case on-time start and on-time finish. This finding indicates that the OR scheduling staff in the study hospital make a concerted effort to schedule the surgical teams with members who have worked well together in the past.
\end{abstract}

Keywords: case on-time start; case on-time finish; perioperative services; team familiarity; OR efficiency

\section{Introduction}

The operating rooms (OR) are the primary source of revenue for many hospitals. However, they are costly to operate and maintain. It is estimated that the cost of an OR procedure is between $\$ 22$ and $\$ 133$ per minute, with an average of $\$ 62$ per minute [1]. Efficient use of the OR would decrease the cost for the patients and the hospitals and thereby improve their bottom lines. Studies have also shown that improved utilization of the OR increase OR staff satisfaction [2,3]. One of the major reasons for OR inefficiencies is surgical cases not starting or finishing on time as scheduled. When a case is delayed, it affects all subsequent scheduled cases such as making them finish late and having staff work overtime [4-7]. Therefore, proper identification of the determinants that affect the surgical cases on-time start and on-time finish in perioperative services can help hospitals develop proper strategies and policies to improve OR efficiency, and thereby, reduce cost. 
Surgical team familiarity in the OR can contribute to reductions in operative time. For example, $\mathrm{Xu}$ et al. [8] reported that after 10 prior collaborations between surgical team members the procedure time in the OR was reduced by 16 minutes on average. Team familiarity can be defined as the number of collaborations between a specific surgeon and a specific perioperative staff member over a given period [9]. While the effect of team familiarity on operative time has been studied [10], how it affects the case on-time start and on-time finish is not fully understood.

This study investigates surgical case scheduling data from a large tertiary referral hospital and academic center to determine why cases start late and/or finish late. It specifically examines how on-time starts and/or on-time finishes are affected by team familiarity and quantifies the impact of team familiarity. Four types of teams are considered in this study: surgeon and anesthesiologist, surgeon and circulating nurse, surgeon and scrub nurse, and surgeon and Certified Registered Nurse Anesthetist (CRNA). Additionally, relevant case information (i.e., number of procedures per case, whether first case, patient condition, types of surgery) are investigated. Two binary logit models are developed: one for case on-time start and one for case on-time finish. For both models, the response variable is whether the case is on-time or late and the explanatory variables consists of different team familiarities and case-related information.

\section{Materials and Methods}

This study did not involve human subjects; therefore, Institutional Review Board approval was not needed.

\subsection{Surgical case data}

This study used surgical case information (without any patient data) from the Greenville Memorial Hospital (GMH) in Greenville, South Carolina for the year 2013. The GMH is a large tertiary referral hospital and academic center. The cases were filtered to retain only the cases which were scheduled to start on regular workdays (i.e., Monday to Friday) and during regularly blocked OR time (i.e., 8 am to $5 \mathrm{pm}$ ). A total of 15,091 cases were in the final data set. These surgeries involved a total of 208 surgeons, 40 anesthesiologists, 115 circulating nurses, 106 scrub nurses and 87 CRNAs. Table 1 presents the descriptive statistics of the explanatory variables considered. Five of the examined explanatory variables are continuous and the rest are binary indicator variables.

Following the work of Maruthappu et al. [9], the surgeon and anesthesiologist team familiarity was defined as the number of times they collaborated during the study period. The mean of 10.82 for the surgeon and anesthesiologist team familiarity indicates that on average a surgeon and an anesthesiologist worked together about 11 times in a year. The other three team familiarity measures were defined in a similar manner. A patient was considered late to OR if the room start time was more than 10 minutes after the scheduled start time. The variable "patient late to OR" was coded as 1 if late, otherwise as 0 . The mean of a binary variable represents the percentage of the

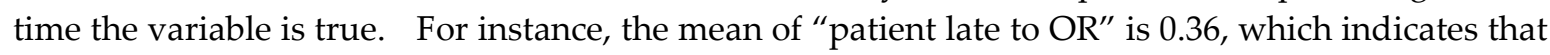
$36 \%$ of the cases that incurred delay was due to getting the patient to the OR.

In this study, for both on-time start and on-time finish, a case was considered to start on-time if the procedure start time was no more than 10 minutes delayed from the scheduled start time and a case was considered to finish late if it finished more than 10 minutes after the scheduled finish time; note that this definition of on-time is different from other hospitals which typically use patient in room time to measure OR on-time performance. Figure 1 shows the percentages of on-time start and on-time finish of surgical cases at GMH in 2013. Note that only about 40 percent of the cases started on-time and about 64 percent of the cases finished on-time. 
Table 1. Descriptive statistics of explanatory variables.

\begin{tabular}{|c|c|c|c|c|c|}
\hline Variable description & Mean & SD & Min & Median & Max \\
\hline Surgeon and Anesthesiologist team familiarity (per year) & 10.82 & 9.13 & 1 & 8 & 56 \\
\hline Surgeon and Circulating Nurse team familiarity (per year) & 17.82 & 22.18 & 1 & 9 & 117 \\
\hline Surgeon and Scrub Nurse team familiarity (per year) & 26.52 & 36.36 & 1 & 11 & 169 \\
\hline Surgeon and CRNA team familiarity (per year) & 8.47 & 12.62 & 1 & 5 & 91 \\
\hline Number of procedures (per case) & 1.71 & 1.11 & 1 & 1 & 14 \\
\hline First case ( 1 if first case, 0 otherwise) & 0.34 & 0.48 & 0 & & 1 \\
\hline \multicolumn{6}{|l|}{ OR billing acuity (proxy for patient condition) } \\
\hline Flat fee and level 0 & 0.01 & 0.09 & 0 & & 1 \\
\hline Level 1 & 0.02 & 0.13 & 0 & & 1 \\
\hline Level 2 & 0.20 & 0.40 & 0 & & 1 \\
\hline Level 3 & 0.32 & 0.46 & 0 & & 1 \\
\hline Level 4 & 0.37 & 0.48 & 0 & & 1 \\
\hline Level 5 & 0.09 & 0.29 & 0 & & 1 \\
\hline Patient late to OR ( 1 if true, 0 otherwise) & 0.36 & 0.48 & 0 & & 1 \\
\hline Emergency surgery ( 1 if true, 0 otherwise) & 0.01 & 0.12 & 0 & & 1 \\
\hline Urgent surgery ( 1 if true, 0 otherwise) & 0.02 & 0.15 & 0 & & 1 \\
\hline
\end{tabular}

Number of observations (i.e., surgical cases) $=15,091$; SD = standard deviation.

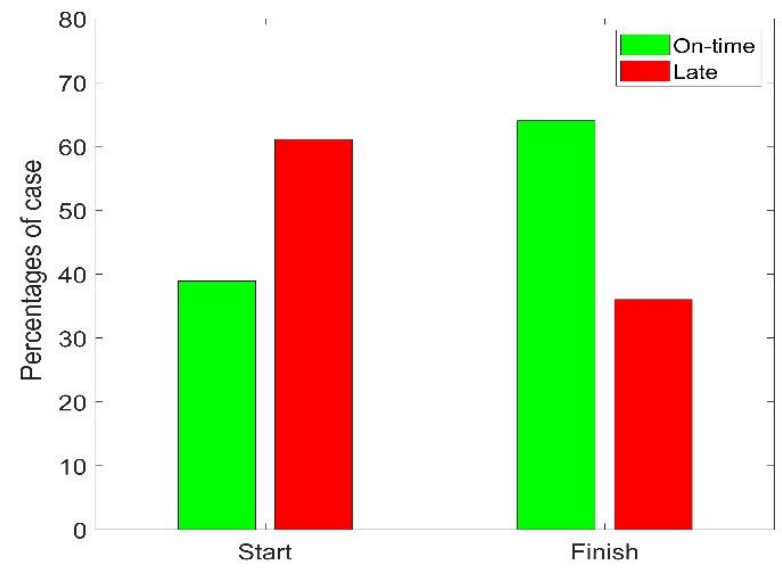

Figure 1. Percentages of GMH surgical case on-time start and on-time finish.

\subsection{Statistical analysis}

Since the response variable considered is binary (i.e., on-time or late), a binary logit model is used, which is a type of discrete choice model. In general, discrete choice models require a choice function $T_{n i}$ that determines the probability of the discrete outcome $i$ (i.e., on-time or late) for surgical case $n$.

$$
T_{n i}=\beta_{i} X_{n i}+\epsilon_{n i}
$$

where $X_{n i}$ is a vector of explanatory variables (see Table 1), $\beta_{i}$ is a vector of parameters to be estimated, and $\epsilon_{n i}$ is a stochastic error term, which is assumed to follow the generalized extreme value distribution. 
For a logit model, the probability $P\left(y_{n i}\right)$ of outcome $i$ for a surgical case $n$ has the following closed form [11]:

$$
P\left(y_{n i}\right)=\frac{\exp \left(\beta_{i} X_{n i}\right)}{1+\exp \left(\beta_{i} X_{n i}\right)}
$$

where $y_{n i}$ is equal to 1 if outcome $i$ (on-time) is observed for surgical case $n$ and 0 otherwise.

Our dataset does not include every variable that contributes to the outcome probability of ontime start and on-time finish and, as a result, can lead to biased estimates and inaccurate inferences. In addition, there is some randomness to perioperative staff scheduling (e.g., anesthesiologist or scrub nurses are typically not scheduled to work every day) that may impact the parameter estimates of the team familiarities. In order to account for the above unobserved variation and randomness (i.e., unobserved heterogeneity) across different surgical cases, mixed (random-parameter) logit models are proposed, which extends the binary logit model by allowing coefficients $\beta$ to be random variables. The distributions of the random variables are described by a set of parameters $\eta$. The mixed logit model can be written as follows [11]:

$$
P_{m}\left(y_{n i}\right)=\int \frac{\exp \left(\beta_{i} X_{n i}\right)}{1+\exp \left(\beta_{i} X_{n i}\right)} f(\beta \mid \eta) d \beta
$$

where $f(\beta \mid \eta)$ denote the probability density function of $\beta$ for a given set $\eta, P_{m}\left(y_{n i}\right)$ denote the total probability of outcome $i$ for a surgical case $n$.

The coefficients $\beta$ allow for case-specific variations of the effect of $X$ on outcome probabilities and the density function $f(\beta \mid \eta)$ is used to determine $\beta$. The mixed logit probabilities are obtained by a weighted average of different values of $\beta$ across cases, where some elements of the vector $\beta$ may be fixed and some may be randomly distributed [12-14]. Any distribution could be adopted for random coefficients $\beta$ in Eq. (3). For that reason, mixed logit models are highly flexible and are able to approximate any random utility model [15]. In this study, we assumed $\beta$ to be normally distributed, which is a widely used assumption [11].

With the above assumption on the distribution of random coefficients $\beta$, the integral in Eq. (3) will not have a closed form. To calculate the outcome probabilities, a simulated maximum likelihood estimation approach is employed. We used 200 Halton draws for the simulation since this number of draws are usually sufficient for accurate parameter estimation [16]. The probability of the outcome is replaced by the corresponding simulated probability obtained from repeated Halton draws.

To assess individual parameter estimates, we used elasticity values which measure the magnitude of the impact of specific variables on the outcome probabilities. Elasticity is computed from the partial derivative for each surgical case $n$ and has the following form [17].

$$
E_{x_{n i k}}^{P_{m}\left(y_{n i}\right)}=\left[1-P_{m}\left(y_{n i}\right)\right] \beta_{i k} x_{n i k}
$$

When the variable $X$ is an indicator variable (i.e., a $0 / 1$ variable), the above derivative approach is not appropriate. Consequently, direct pseudo-elasticities are calculated as the percentage change in probability when an indicator variable is switched from 0 to 1 or from 1 to 0 [17].

$$
E_{x_{n i k}}^{P_{m}\left(y_{n i}\right)}=\frac{P_{m}\left(y_{n i}\right)\left[\text { given } x_{n i k}=1\right]-P_{m}\left(y_{n i}\right) \text { [given } x_{n i k}=0 \text { ] }}{\left.P_{m}\left(y_{n i}\right) \text { [given } x_{n i k}=0\right]}
$$

where $x_{\text {nik }}$ is the $k$ th explanatory variable associated with outcome $i$ for surgical case $n$. The direct pseudo-elasticity was calculated for outcome $i$ and each case $n$; and hence, the average direct pseudo-elasticity for outcome $i$ is computed as the average over the entire sample of surgical cases.

Following the above modeling framework, two random parameter binary logit models (i.e., mixed logit model) were estimated, one for surgical case on-time start and one for on-time finish where the outcome is either on-time or late. The econometric software NLOGIT version 5.0 was used for the model estimation, and variables were retained in the model specification if they had $t$ statistics corresponding to the $90 \%$ confidence level or higher on a two-tailed $t$-test. The random 
parameters were retained if their standard deviations had $t$-statistics corresponding to the $90 \%$ confidence level or higher. The binary logit model estimation results for the surgical case on-time start is presented in Table 2 and results for the case on-time finish is presented in Table 3. In both tables, the elasticity values for the factors are shown.

Table 2. Binary logit model results for the surgical case on-time start.

\begin{tabular}{lcccc}
\hline Parameter & Coefficient & z-stat & P-value & Elasticity \\
\hline $\begin{array}{l}\text { Surgeon \& Anesthesiologist team familiarity (SD } \\
\text { of random parameter) }\end{array}$ & $0.017(0.086)$ & $8.17(38.11)$ & $0.000(0.000)$ & $11.1 \%$ \\
$\begin{array}{l}\text { Surgeon \& Circulating Nurse team familiarity } \\
\text { (SD of random parameter) }\end{array}$ & $0.001(0.006)$ & $1.76(7.56)$ & $0.079(0.000)$ & $1.6 \%$ \\
Surgeon \& Scrub Nurse team familiarity (SD of & & & & \\
random parameter) & $0.001(0.001)$ & $1.76(2.21)$ & $0.078(0.027)$ & $1.3 \%$ \\
Surgeon \& CRNA team familiarity (SD of & & & & \\
random parameter) & $0.009(0.137)$ & $4.16(38.52)$ & $0.000(0.000)$ & $4.5 \%$ \\
Number of procedures & -0.059 & -4.14 & 0.000 & $-6.1 \%$ \\
First case & 0.781 & 25.45 & 0.000 & $16.1 \%$ \\
OR billing acuity & & & & \\
$\quad$ Level 1 & -0.329 & -2.77 & 0.006 & $-0.3 \%$ \\
$\quad$ Level 2 & -0.586 & -14.21 & 0.000 & $-7.0 \%$ \\
$\quad$ Level 3 & -0.755 & -18.15 & 0.000 & $-14.2 \%$ \\
$\quad$ Level 4 & -0.991 & -24.47 & 0.000 & $-21.9 \%$ \\
$\quad$ Level 5 & -1.754 & -22.31 & 0.000 & $-9.7 \%$ \\
Emergency surgery & 0.662 & 5.98 & 0.000 & $0.6 \%$ \\
Number of observations & 15,091 & & & \\
Chi-squared $4 d f$ ) & 74.654 & & & \\
Log-likelihood at convergence & $-9,573.041$ & & & \\
Akaike Information Criterion (AIC) & $19,178.1$ & & & \\
\hline
\end{tabular}

$\mathrm{SD}=$ standard deviation; $d f=$ degree of freedom.

\section{Results}

For the on-time start model, four parameters are treated as random (vary across the population) since their standard deviations are statistically significant (see the z-statistics of the first four parameters in Table 2). The other parameters are treated as fixed since their standard deviations are not significantly different from zero. The parameter for surgeon and anesthesiologist team familiarity was found to be random and normally distributed with a mean of 0.017 and standard deviation (SD) of 0.086 . Given these estimates, $42.2 \%$ of the surgical cases have parameter values less than 0 and $57.8 \%$ greater than 0 . This implies that less than half of the surgical cases result in a decrease in case on-time start and more than half result in an increase in on-time start. The parameters for the surgeon and circulating nurse team familiarity, surgeon and scrub nurse team familiarity, and surgeon and CRNA team familiarity were also found to be random and normally distributed. For surgeon and circulating nurse team familiarity, $43.4 \%$ of the surgical cases have parameters values less than 0 and $56.6 \%$ greater than 0 (mean $=0.001$ and $\mathrm{SD}=0.006$ ). Thus, for less than half of the surgical cases, higher surgeon and circulating nurse team familiarity decreases the likelihood of case on-time start, for more than half it increases the likelihood of on-time start. For 
surgeon and scrub nurse team familiarity, $15.9 \%$ of the surgical cases have parameters values less than 0 and $84.1 \%$ greater than 0 (mean $=0.001$ and $S D=0.001)$. This implies that in a small proportion of the surgical cases, the surgeon and scrub nurse team familiarity decreases the likelihood of case on-time start, while in a majority of surgical cases, the likelihood increases. Lastly, the parameter for surgeon and CRNA team familiarity is random with mean 0.009 and SD 0.137. With these estimates, for $47.4 \%$ of the surgical cases have parameters values less than 0 and $52.6 \%$ greater than 0 . Thus, for slightly less than half of the surgical cases result in a decrease in case on-time start and slightly more than half result in an increase in on-time start.

Table 3. Binary logit model results for the surgical case on-time finish.

\begin{tabular}{lcccc}
\hline Parameter & Coefficient & z-stat & P-value & Elasticity \\
\hline $\begin{array}{l}\text { Surgeon \& Circulating Nurse team familiarity } \\
\text { (SD of random parameter) }\end{array}$ & $0.004(0.025)$ & $3.04(19.50)$ & $0.002(0.000)$ & $4.3 \%$ \\
$\begin{array}{l}\text { Surgeon \& Scrub Nurse team familiarity (SD of } \\
\text { random parameter) }\end{array}$ & $0.007(0.011)$ & $8.31(13.87)$ & $0.000(0.000)$ & $11.6 \%$ \\
Surgeon \& CRNA team familiarity (SD of & & & & \\
random parameter) & $0.019(0.052)$ & $8.26(18.22)$ & $0.000(0.000)$ & $10.3 \%$ \\
Number of procedures & -0.168 & -10.15 & 0.000 & $-18.1 \%$ \\
First case (SD of random parameter) & $-0.017(0.775)$ & $-0.35(16.11)$ & $0.725(0.000)$ & $-0.4 \%$ \\
OR billing acuity & & & & \\
$\quad$ Level 1 & 3.392 & 10.78 & 0.000 & $3.5 \%$ \\
$\quad$ Level 2 & 2.689 & 30.21 & 0.000 & $33.8 \%$ \\
$\quad$ Level 3 & 1.785 & 29.61 & 0.000 & $35.5 \%$ \\
$\quad$ Level 4 & 1.261 & 23.43 & 0.000 & $29.4 \%$ \\
$\quad$ Level 5 & 0.423 & 4.82 & 0.000 & $2.5 \%$ \\
Urgent surgery & -0.412 & -2.67 & 0.008 & $-0.6 \%$ \\
Patient late to OR (SD of random parameter) & $-6.416(9.485)$ & $-36.97(28.80)$ & $0.000(0.000)$ & $-145.4 \%$ \\
Number of observations & 15,091 & & & \\
Chi-squared (5 df) & 221.304 & & & \\
Log-likelihood at convergence & $-6,585.781$ & & & \\
Akaike Information Criterion (AIC) & $13,205.6$ & & & \\
\hline
\end{tabular}

$\mathrm{SD}=$ standard deviation; $d f=$ degree of freedom.

For the case on-time finish model, five of the parameters were found to be random and normally distributed (Table 3). The parameter for surgeon and circulating nurse team familiarity is random with a mean of 0.004 and SD of 0.025 . That means for $43.6 \%$ of the surgical cases have parameters values less than 0 and $56.4 \%$ greater than 0 . Thus, for less than half of the surgical cases, surgeon and circulating nurse team familiarity decreases the likelihood of case on-time finish, for more than half it increases the likelihood of on-time finish. In case of surgeon and scrub nurse team familiarity, for $26.2 \%$ of the surgical cases have parameters values less than 0 and $73.8 \%$ greater than 0 (mean = 0.007 and $S D=0.011$ ). For the majority of the surgical cases, surgeon and scrub nurse team familiarity increases the likelihood of case on-time finish. Lastly, for surgeon and CRNA team familiarity 35.7 of the surgical cases have parameters value less than 0 and $64.3 \%$ greater than 0 . For the majority of the cases, surgeon and CRNA team familiarity increases the likelihood of on-time finish. The parameter for first case is random with mean -0.017 and SD 0.775. Given these estimates, $50.9 \%$ of the surgical case have parameters value less than 0 and rest of the cases have 
greater than 0 . This implies that about half of the cases being first case is associated with increased probability of case finished on-time. The parameter for patient late to OR is random with mean 6.416 and SD 9.485. The estimates suggest that for $75.1 \%$ of the surgical cases have parameter values less than 0 and for the rest, it is greater than 0 . Thus, for majority of the cases patient being late to OR decreases the likelihood of on-time finish.

\section{Discussion}

This study examined team familiarity's influence on perioperative performance with a different focus than Maruthappu et al. [9], but it obtained similar conclusions. Specifically, this study found that increased familiarity both increases on time starts and reduces operative time. Team familiarity is manageable and should be considered by hospital administrators and operating room managers when possible to do so. This practice currently occurs in the study hospital (GMH) where specialties have dedicated teams (e.g., heart teams and orthopedic trauma teams), and although these teams were created to enhance the quality mission, they also enhance the communication between team members, resulting in higher team familiarity.

The positive coefficients of the parameters regarding patient levels indicate that higher acuity levels increase the probability of the case finishing on time compared to "flat fee and level 0." A possible explanation for this finding is that the average case time being used to schedule cases increases as the patient acuity increases; at GMH, the average case time is based on the last 10 similar cases where the highest and lowest case time are not considered (known as the Olympic average). Moreover, it is possible that the surgeon could have provided a longer case time to the scheduling office. The negative coefficient of the parameter for urgent surgery suggests that the likelihood of a case finishing on-time decreases if the case is an urgent surgery. However, the elasticity indicates this probability is very low $(0.6 \%)$. The elasticity value indicates that when a patient late to the OR, the likelihood of a case finishing on time decreases by 145.4 percent. This value is the highest among all factors considered for the case on-time finish.

The random parameter for the surgeon and anesthesiologist team familiarity captures the unobserved heterogeneity across different surgical cases. Table 4 summarizes the change in on-time start and finish in terms of elasticity values for selected factors. The elasticity suggests that a one percent increase in surgeon and anesthesiologist team familiarity increases the likelihood of the surgical case starting on time by $11.1 \%$ (95\% confidence interval (CI): $8.4 \%-13.8 \%$ ). Similarly, it is found that a one percent increase in surgeon and circulating nurse team familiarity increase the likelihood of the case starting on time by $1.6 \%$ (CI: $0.2 \%-3.4 \%$ ). For the surgeon and scrub nurse team, a one percent increase in familiarity increases the case on time start by $1.3 \%$ (CI: $0.1 \%-2.7 \%$ ), and for the surgeon and CRNA team, the increase in on-time start is $4.5 \%$ (CI: $2.4 \%-6.6 \%$ ).

The negative parameter for number of procedures indicates that as the number of procedures for a case increases the probability of an on-time start will decrease. Specifically, a one percent increase in the number of procedures decreases the likelihood of a case starting on time by $6.1 \%$ (CI: $3.2 \%-8.9 \%)$.

The indicator variable first case has a positive coefficient, which suggests that the first case has an increased likelihood of starting on-time (16.1\%, CI: $14.9 \%-17.3 \%)$. In regard to patient condition, compared to level 0, other levels decrease the likelihood of the case starting on time. Lastly, the likelihood of a case starting on time increases if the case is an emergency surgery; however, this likelihood is not very high (only $0.6 \%$ ).

A one percent increase in the surgeon and circulating nurse team familiarity increases the likelihood of a surgical case finishing on time by $4.3 \%$ (CI: $1.5 \%-7.1 \%$ ). For the surgeon and scrub nurse team, a one percent increase in familiarity increases the probability of a case finishing on time by $11.6 \%$ (CI: $8.9 \%-14.3 \%$ ), and for the surgeon and CRNA team, the increase in likelihood of finishing on time is $10.3 \%$ (CI: $7.9 \%-12.7 \%$ ). 
Table 4. Change in on-time start and finish for selected factors.

\begin{tabular}{lccc}
\hline Variable & Change & On-time start change (CI) & On-time finish change (CI) \\
\hline $\begin{array}{l}\text { Surgeon \& Anesthesiologist team } \\
\text { familiarity }\end{array}$ & $+1 \%$ & $+11.1 \%(+8.4 \%$ to $13.8 \%)$ & \\
$\begin{array}{l}\text { Surgeon \& Circulating Nurse team } \\
\text { familiarity }\end{array}$ & $+1 \%$ & $+1.6 \%(+0.2 \%$ to $3.4 \%)$ & $+4.3 \%(+1.5 \%$ to $7.1 \%)$ \\
$\begin{array}{l}\text { Surgeon \& Scrub Nurse team } \\
\text { familiarity }\end{array}$ & $+1 \%$ & $+1.3 \%(+0.1 \%$ to $2.7 \%)$ & $+11.6 \%(+8.9 \%$ to $+14.3 \%)$ \\
$\begin{array}{l}\text { Surgeon \& CRNA team familiarity } \\
\text { First Case }\end{array}$ & $+1 \%$ & $+4.5 \%(+2.4 \%$ to $6.6 \%)$ & $+10.3 \%(+7.9 \%$ to $+12.7 \%)$ \\
Number of Procedures in case & $+1 \%$ & $-16.1 \%(+14.9 \%$ to $+17.3 \%)$ & $-0.4 \%(-2.4 \%$ to $+1.7 \%)$ \\
Patient Late to OR & Yes & & $-18.1 \%(-21.6 \%$ to $-14.6 \%)$ \\
\hline
\end{tabular}

$\mathrm{CI}=95 \%$ Confidence Interval.

The coefficient of the number of procedures is negative, which suggests that as the number of procedures for a case increases the likelihood of the case finishing on time decreases. A one percent increase in number of procedures decreases the likelihood of a case finishing on-time by $18.1 \%$ (CI: $14.6 \%-21.6 \%)$.

\subsection{Limitations}

Our study has several limitations which should be considered when applying the findings. First, it uses surgical case data from a single hospital and the data was available for only one year. Second, the cases were limited to only those performed during regular weekdays and blocked OR times. The findings would be more generalizable if the dataset had cases from multiple hospitals, spanning over several years, and from all work hours.

\section{Conclusions}

The purpose of this work was to determine the significant factors and their impact on the ontime start and on-time finish. Special attention was given to the impact of different surgical team familiarities. It was found that increased familiarity between the surgeon and anesthesiologist, the surgeon and circulating nurse, the surgeon and scrub nurse, and the surgeon and CRNA improved the likelihood of OR cases starting on time and finishing on time. The findings from this study suggest that hospitals should consider team familiarity when scheduling OR staff. Also, it should put priority on getting patients to the OR on time.

Author Contributions: Conceptualization, M.U., L.F., N.H., and K.T.; methodology, M.U. and N.H.; formal analysis, M.U.; writing-original draft preparation, M.U.; writing - review and editing, M.U., L.F., N.H., K.T., and R.A. All authors have read and agreed to the published version of the manuscript.

Funding: This research received no external funding.

Acknowledgments: We would like to acknowledge Prisma Health (formerly Greenville Health System) for their support in providing data for analysis.

Conflicts of Interest: The authors declare no conflict of interest.

\section{References}

1. Macario, A. What does one minute of operating room time cost? Journal of Clinical Anesthesia 2010, 22, 233-236, doi:10.1016/j.jclinane.2010.02.003. 
2. Wachtel, R.E.; Dexter, F. Influence of the operating room schedule on tardiness from scheduled start times. Anesthesia \& Analgesia 2009, 108, 1889-1901, doi:10.1213/ane.0b013e31819f9f0c.

3. Wachtel, R.E.; Dexter, F. Reducing tardiness from scheduled start times by making adjustments to the operating room schedule. Anesthesia $\mathcal{E}$ Analgesia 2009, 108, 1902-1909, doi:10.1213/ane.0b013e31819f9fd2.

4. van Veen-Berkx, E.; Elkhuizen, S.G.; Kalkman, C.J.; Buhre, W.F.; Kazemier, G. Successful interventions to reduce first-case tardiness in Dutch university medical centers: results of a nationwide operating room benchmark study. The American Journal of Surgery 2014, 207, 949-959, doi:10.1016/j.amjsurg.2013.09.025.

5. Dexter, F.; Epstein, R.H.; Schwenk, E.S. Tardiness of starts of surgical cases is not substantively greater when the preceding surgeon in an operating room is of a different versus the same specialty. Journal of Clinical Anesthesia 2019, 53, 20-26, doi:10.1016/j.jclinane.2018.09.027.

6. $\quad$ Ernst, C.; Szczesny, A.; Soderstrom, N.; Siegmund, F.; Schleppers, A. Success of commonly used operating room management tools in reducing tardiness of first case of the day starts: evidence from German hospitals. Anesthesia \& Analgesia 2012, 115, 671-677, doi:10.1213/ANE.0b013e31825c0486.

7. Martin, L.; Langell, J. Improving on-time surgical starts: the impact of implementing pre-OR timeouts and performance pay. Journal of Surgical Research 2017, 219, 222-225, doi:10.1016/j.jss.2017.05.092.

8. Xu, R.; Carty, M.J.; Orgill, D.P.; Lipsitz, S.R.; Duclos, A. The teaming curve: a longitudinal study of the influence of surgical team familiarity on operative time. Annals of surgery 2013, 258, 953-957, doi:10.1097/SLA.0b013e3182864ffe.

9. Maruthappu, M.; Duclos, A.; Zhou, C.D.; Lipsitz, S.R.; Wright, J.; Orgill, D.; Carty, M.J. The impact of team familiarity and surgical experience on operative efficiency: a retrospective analysis. Journal of the Royal Society of Medicine 2016, 109, 147-153, doi:10.1177/0141076816634317.

10. Xiao, Y.; Jones, A.; Zhang, B.B.; Bennett, M.; Mears, S.C.; Mabrey, J.D.; Kennerly, D. Team consistency and occurrences of prolonged operative time, prolonged hospital stay, and hospital readmission: a retrospective analysis. World Journal of Surgery 2015, 39, 890-896, doi:10.1007/s00268-014-2866-7.

11. Train, K.E. Discrete Choice Methods with Simulation, 2nd ed.; Cambridge University Press: New York, NY, 2009.

12. Uddin, M.; Huynh, N. Truck-involved crashes injury severity analysis for different lighting conditions on rural and urban roadways. Accident Analysis $\mathcal{E}$ Prevention 2017, 108, 44-55, doi:10.1016/j.aap.2017.08.009.

13. Uddin, M.; Huynh, N. Injury severity analysis of truck-involved crashes under different weather conditions. Accident Analysis \& Prevention 2020, 141, 105529, doi:10.1016/j.aap.2020.105529.

14. Uddin, M.; Ahmed, F. Pedestrian injury severity analysis in motor vehicle crashes in Ohio. Safety 2018, 4, 20, doi:10.3390/safety4020020.

15. McFadden, D.; Train, K. Mixed MNL models for discrete response. Journal of Applied Econometrics 2000, 15, 447-470, doi:10.1002/1099-1255(200009/10)15:5<447::AID-JAE570>3.0.CO;2-1.

16. Uddin, M.; Huynh, N. Factors influencing injury severity of crashes involving HAZMAT trucks. International journal of transportation science and technology 2018, 7, 1-9, doi:10.1016/j.jitst.2017.06.004.

17. Washington, S.; Karlaftis, M.G.; Mannering, F.; Anastasopoulos, P. Statistical and Econometric Methods for Transportation Data Analysis, 3rd ed.; Chapman and Hall/CRC: Boca Raton, FL, 2020. 